TUMOUR MICROENVIRONMENT

\section{Nervous tumours}

a novel
crosstalk
between the
brain and
prostate
tumours

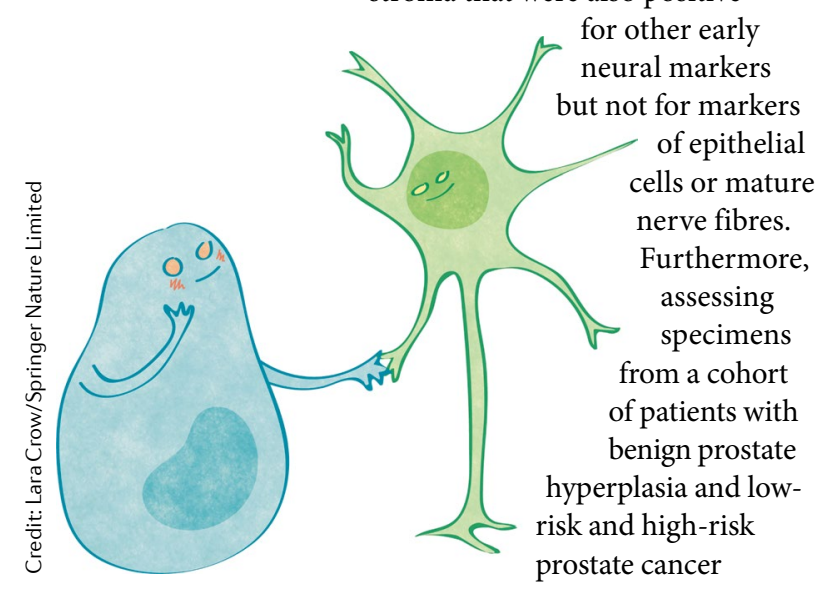

Autonomic nerve fibres present

in the tumour microenvironment

(TME) have been shown to

influence the progression of several types of cancer. In particular, the presence of an expanded number of neurons per ganglia in human prostate tumours has hinted at the possibility that, in this cancer type at least, neurogenesis - the de novo generation of functional neurons from neural precursors - could be occurring to support tumour initiation and dissemination. However, until now the origins of these newly formed nerve fibres have not been well documented. Providing one possible explanation, a team led by Claire Magnon has revealed that neural progenitors arising from the central nervous system (CNS) in mice can migrate to and infiltrate primary prostate tumours and metastases, where they differentiate into new adrenergic neurons to regulate the early stages of tumour development.

First, the authors set out to establish whether neural progenitors were present in human prostate tumours. Using doublecortin (DCX) as a standard marker of neural progenitors localized in the developing and adult neurogenic regions of the CNS, immunohistochemical staining of human primary prostate adenocarcinomas demonstrated the presence of $\mathrm{DCX}^{+}$cells in the stroma that were also positive showed that the density of $\mathrm{DCX}^{+}$ cells correlated with tumour aggressiveness, invasion and recurrence.

Investigating this phenomenon further in a transgenic mouse model of prostate cancer, the authors expressed human MYC from the rat probasin promoter exclusively in prostate epithelial cells to generate Hi-MYC mice. To monitor DCX ${ }^{+}$ cells in tumours, enhanced yellow fluorescent protein (eYFP) was inducibly expressed under the control of the human $D C X$ promoter. Serving as a positive control, DCX-eYFP ${ }^{+}$cells were found in the neurogenic regions of the brain (the subventricular zone (SVZ), olfactory bulbs and dentate gyrus). Within prostate tumours, these cells were localized in the stroma.

Isolation and further

characterization of the tumour-associated DCX-eYFP ${ }^{+}$ cells revealed that they were lineage marker negative and did not arise from tumour cells, but they did express SCA1, a marker of prostate stromal cells, and markers of neural progenitors. In addition, these same cells were not found in healthy prostate tissues of control mice without MYC transgene expression.

To determine whether the tumour-associated neural progenitor cells could differentiate into neurons in vivo, the authors performed genetic tracing experiments. In the Hi-MYC model, prostate tumours develop from prostate intraepithelial neoplasia (PIN) approximately 12 weeks after birth. Addition of tamoxifen at 3 weeks after birth saw DCX-eYFP ${ }^{+}$ cells emerge in prostate tumours at 8,12 and 16 weeks but not in healthy tissues adjacent to the prostate. At 8 months after birth, $\mathrm{eYFP}^{+}$neuroblasts gave rise to $\mathrm{eFP}^{+}$nerve fibres that expressed tyrosine hydroxylase, indicative of new adrenergic neurons originating in situ in the TME from neural progenitors.

Oscillations in the numbers of DCX-eYFP $^{+}$cells in the SVZ were noted at 6 weeks after birth and correlated with DCX-eYFP ${ }^{+}$cells detected in the blood of 6,12 and 16-week-old mice, suggesting egress of neural progenitors from the SVZ and migration via the bloodstream towards prostate tumours. In support of this, stereotaxic injection of a tdTomato-expressing lentiviral vector into the SVZ of Hi-MYC mice led to SFP $^{+}$tdTomato $^{+}$cells in the circulation and prostate tumours but not in healthy tissues in the vicinity of the tumours. Interestingly, the vascular permeability of the blood-brain barrier (BBB) close to the SVZ was disrupted, indicative of an 'escape route'. Similar stereotaxic injection of the tdTomato lentiviral vector into the dentate gyrus did not result in $\mathrm{eYFP}^{+}$tdTomato $^{+}$cells within tumours or a breach of the $\mathrm{BBB}$ within this neurogenic region. However, the tumour-derived factors that drive the selective migration remain undetermined.

tdTomato ${ }^{+}$cells were also identified in tumours formed in another transgenic prostate cancer mouse model driven by Pten deletion, as well as in the MMTV-PyMT transgenic breast cancer mouse model following stereotaxic injection into the SVZ, demonstrating that this might be a common feature of early tumours. Furthermore, neural progenitors arising from the SVZ also infiltrated primary tumours and metastatic tissues of mice bearing orthotopic xenografts of human PC3 prostate cancer cells. Finally, the importance of neural progenitors in the initial stages of tumour formation was underscored by the observation that specific genetic depletion of $\mathrm{DCX}^{+}$ cells from Hi-MYC mice decreased the incidence of PIN lesions.

This study has uncovered a novel crosstalk between the brain and prostate tumours, whereby tumours can selectively recruit cells from distant organs through systemic communication to fulfil requirements for growth and dissemination. However, whether the same mechanism driving the generation of new neurons in tumours operates in humans will need to be established, especially in light of recent controversy over the extent and sites of adult neurogenesis.

Anna Dart

ORIGINAL ARTICLE Mauffrey, P. et al. Progenitors from the central nervous system drive neurogenesis in cancer. Nature https://doi.org/10.1038/s41586019-1219-y (2019) 